\section{Nasogastric tube insertion easily done: The SORT maneuver}

Sir,

Nasogastric tube (NGT) insertion in anesthetized and/or critically ill patients who cannot swallow is a challenging procedure. The anatomic structure of the pharynx and larynx halts easy passage of NGT into the esophagus. SORT maneuver for NGT insertion has been proposed recently as a new way to facilitate this and prevents from inadvertent trauma to the patient. This is the first case report on successful usage of SORT maneuver.

A 61-year-old opium addicted man who underwent coronary artery bypass graft surgery for severe three vessel, received $40 \mathrm{mg}$ morphine sulfate during the operation to control pain and stabilize hemodynamic indices. At the end of surgery, a NGT was inserted to give medications to handle probable episodes of patient's pain, tachycardia, and hypertension. In this patient, SORT maneuver was applied for NGT insertion, ${ }^{[1]}$ to avoid brisk hemodynamic responses [Video 1].

NGT insertion is a common procedure implemented in patients with wide ranges of diseases and conditions from very healthy subjects undergoing elective surgeries to critically ill intubated patients. Despite being simple to perform in awake patients, it is often challenging to insert NGT in intubated patients in which unconsciousness halts the ability of swallowing.

Being softer and less traumatic than previous types, the polyurethane NGTs are broadly used in our daily practice. This advantage comes with its own disadvantages. Becoming softer on exposure to body 


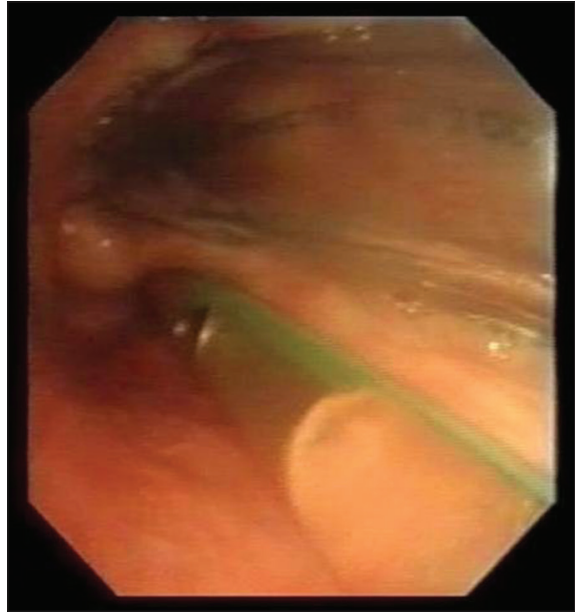

Figure I: Endoscopic view of nasogastric tube insertion using SORT maneuver

temperature, NGTs are more prone to kink; this in turn leads to unsuccessful NGT insertion. ${ }^{[2]}$ Other NGT related factors contributing to unsuccessful NGT insertion are the following: (1) Several nonopposing lateral distal eyes causing NGT kink, (2) curved NGT, when in the packet, encouraging NGT coiling while entering the mouth, and (3) endotracheal tube's inflated balloon in intubated patients obstructing NGT passage. ${ }^{[2,3]}$

Anatomical factors also immensely contribute to unsuccessful NGT insertion. Piriform sinus and arytenoid cartilages are the most common places wherein NGT is usually lodged. ${ }^{[4]}$ Numerous NGT insertion techniques have been introduced to overcome these problems; including reverse guide wire-assisted techniques, neck flexion with lateral neck pressure, and Sellick's maneuver. ${ }^{[5]}$ SORT maneuver would be a reasonable approach to address these concerns. SORT is mnemonic for the four main steps of the maneuver: Sniffing position, NGT orientation, contralateral rotation, and twisting movement.

After the patient is placed in sniffing position, NGT is oriented from nose to esophagus entrance based on anatomy. It should be taken into consideration that no force should be applied against any resistance while insertion [Figure 1]. We should change the position of NGT tip by back and forth and rotational movements until it find its way through esophagus without resistance. Sliding distal end of NGT on posterior wall of oropharynx into esophagus by tip of index finger is sometimes helpful for correct orientation. Rotation of head to contralateral side (of NGT entrance) is very important step; this blunts the ipsilateral piriform sinus while sniffing position thrusts arytenoid cartilage away from esophageal entrance. Then, NGT tip can be directed deep into esophagus by twisting movements to reduce resistance. External pressure on piriform sinus area might be applied if the initial maneuver fails. This maneuver could also be of assistance in transesophageal echocardiography probe insertion.

\section{Financial support and sponsorship Nil.}

\section{Conflicts of interest}

There are no conflicts of interest.

Mahdi Najafi
Tehran Heart Center, Tehran University of Medical Sciences, Tehran, Iran

Correspondence: Dr. Mahdi Najafi, Tehran Heart Center, Tehran University of Medical Sciences, North Karegar Avenue, 1411713138 Tehran, Iran. E-mail: najafik@sina.tums.ac.ir

\section{References}

1. Najafi M, Golzari SE. SORT maneuver for nasogastric tube insertion. Anaesthesia 2016;71:351.

2. Tsai YF, Luo CF, Illias A, Lin CC, Yu HP. Nasogastric tube insertion in anesthetized and intubated patients: A new and reliable method. BMC Gastroenterol 2012;12:99.

3. Dali JS, Gombar KK, Kumar P, Anand R, Seshadri TR. Nasogastric tube placement in difficult cases: A novel and simple maneuver. J Anaesthesiol Clin Pharmacol 2005;21:429-34

4. Ozer S, Benumof JL. Oro- and nasogastric tube passage in intubated patients: Fiberoptic description of where they go at the laryngeal level and how to make them enter the esophagus. Anesthesiology 1999;91:137-43.

5. Mandal MC, Dolai S, Ghosh S, Mistri PK, Roy R, Basu SR, et al. Comparison of four techniques of nasogastric tube insertion in anaesthetised, intubated patients: A randomized controlled trial. Indian J Anaesth 2014;58:714-8.

This is an open access article distributed under the terms of the Creative Commons Attribution-NonCommercial-ShareAlike 3.0 License, which allows others to remix, tweak, and build upon the work non-commercially, as long as the author is credited and the new creations are licensed under the identical terms.

\section{Video Available on: www.jiccm.org}

\begin{tabular}{|l|l|}
\hline \multicolumn{2}{|c|}{ Access this article online } \\
\hline Quick Response Code: & Website: \\
\hline & www.ijccm.org \\
\cline { 2 - 2 } & Dol: 10.4103/0972-5229.188214 \\
\hline
\end{tabular}

How to cite this article: Najafi M. Nasogastric tube insertion easily done: The SORT maneuver. Indian J Crit Care Med 2016;20:492-3. 